\title{
Dynamic morphology of the secundum atrial septal defect evaluated by three dimensional transoesophageal echocardiography
}

\author{
Y V Maeno, L N Benson, P R McLaughlin, C Boutin
}

\begin{abstract}
Objective-To define by three dimensional echocardiography the pattern and potential determinants of contraction of a secundum atrial septal defect through the cardiac cycle, and to evaluate the possibility of using cross sectional transthoracic and transoesophageal imaging to assess the dynamic nature of the defect.

Design-Three dimensional echocardiography was performed using a multiplane transoesophageal probe on 50 patients with a secundum atrial septal defect (median age 9.8 years). Nine patients were excluded because of poor images or morphological features that precluded defect measurement. In 41 cases, defect area, long and short axis length, and distance of the attenuated anterior rim were measured in their largest and smallest dimensions.

Results-Defect area changed significantly through the cardiac cycle (mean change $61 \%$, $\mathrm{p}<0.0001$; range $17 \%$ to $86 \%$ ). The defect contracted symmetrically and was not related to patient age, defect size, heart rate, Qp/Qs ratio, the presence of an aneurysmal atrial septum, or attenuated anterior rim. In all cases with an attenuated anterior rim $(n=13)$, the length of the rim significantly decreased $(p=0.001)$ during atrial systole. Dynamic changes measured by either transthoracic or transoesophageal cross sectional images did not correlate with those obtained by three dimensional imaging.

Conclusions-Three dimensional echocardiography shows dynamic features of defects in the atrial septum. This information may lead to an improved understanding of the pathophysiology of atrial shunting.

(Heart 2000;83:673-677)
\end{abstract}

Keywords: echocardiography; atrial septal defect; congenital heart disease

A secundum atrial septal defect is one of the more common cardiac defects occurring in isolation, and accounts for between $5 \%$ and $15 \%$ of all congenital heart malformations. ${ }^{1}$ Morphological studies have shown variable features in size, position, and shape, ${ }^{23}$ but the relation between these features and clinical outcome seems variable. ${ }^{4}$ The development of new techniques of closure, including minimal access surgery ${ }^{5}$ and transcatheter occlusion, ${ }^{7-11}$ makes it even more difficult to decide upon the most appropriate treatment for any particular patient. ${ }^{12-14}$

It has recently been shown that dynamic three dimensional echocardiography can reveal changes in the size of an atrial septal defect during the cardiac cycle, the extent of which varies from patient to patient. ${ }^{15}{ }^{16}$ Further investigation of the dynamic nature of the atrial septal defect, especially in relation to the variability of contraction, may improve our understanding of the clinical features of these lesions and enable us to select the most appropriate treatment. Our aim in this study was threefold: first, to define by three dimensional echocardiography the pattern of contraction in the atrial septal defect through the cardiac cycle; second, to define the potential determinants of such changes; and third, to compare cross sectional transthoracic and transoesophageal echocardiography with three dimensional information, to see whether cross sectional images could be used to assess the dynamic nature of these defects.

\section{Methods}

PATIENT POPULATION

Between November 1996 and January 1998, three dimensional echocardiographic images of atrial septal defects were reconstructed in 50 patients who were undergoing transoesophageal echocardiography at either the Hospital for Sick Children, Toronto, or the Toronto Hospital, Toronto General Division. From this group, 41 patients were included in this study to assess the dynamic nature of the defect. Morphological features precluded measurement in five patients, three with multiple fenestrations and two having a gap between the septum primum and the septum secundum. The remaining four were excluded because of poor three dimensional images from either motion artefact or inadequate cross sectional imaging during acquisition. The age of the patients ranged from 4 to 72 years, median 10 years. Heart rate at the time of three dimensional echocardiography was available in 36 patients and ranged from 60 to 142 beats/min, median 87. The ratio of pulmonary to systemic flow $(\mathrm{Qp} / \mathrm{Qs})$ measured at cardiac catheterisation was available in 27 patients, and ranged from 1.0 to $4.6: 1$, median $1.8: 1$.

\section{THREE DIMENSIONAL ECHOCARDIOGRAPHY}

All patients underwent transoesophageal echocardiography, using Hewlett-Packard Sonos 2500 hardware and software revision B.2 (Hewlett-Packard Inc, Andover, Massachu- 

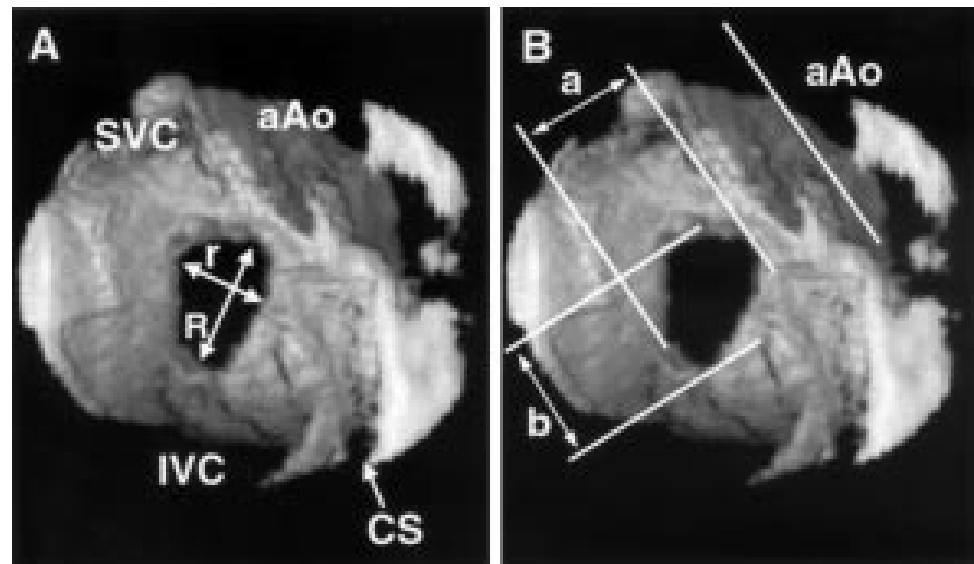

Figure 1 Measurement of the size of atrial septal defects. Dimension of major axis $(R)$ and minor axis $(r)$ (panel $A$ ). Length of defect, perpendicular (a) and parallel $(b)$ to the ascending aorta ( $(A A)$ ) (panel $B)$. CS, coronary sinus; IVC, inferior vena cava; SVC, superior vena cava.

setts, USA), which has the capability for acquisition of serial cross sectional echocardiographic images by a multiplane transoesophageal probe. Under general anaesthesia or conscious sedation, serial cross sectional images with increments of $3^{\circ}$ were acquired, using a $6.2 / 5.0 \mathrm{MHz}$ or a $5.0 / 3.7$ $\mathrm{MHz}$ probe with cardiac and respiratory cycle gating. In all 41 patients, a single investigation was attempted to provide a dataset for three dimensional reconstruction. The stored serial cross sectional data in the optical disk were transferred to a computer (Echo-Scan, version 3.1, TomTec Imaging Systems, Inc, Boulder, Colorado, USA) for analysis of the three dimensional echocardiographic dataset, as previously described. ${ }^{17-19}$ Volume rendered images with a single still frame or with dynamic motion, and a cross sectional image in an optimal cutting plane using "any plane" function were obtained from these three dimensional datasets for further measurement.

\section{MEASUREMENTS AND CALCULATIONS}

From dynamic, volume rendered images looking at the defect from the right atrium, two specific frames-the largest and the smallest dimension during the cardiac cycle-were defined, and areas were calculated using the following method. Once the angles of the major and minor axes of the defect were defined on
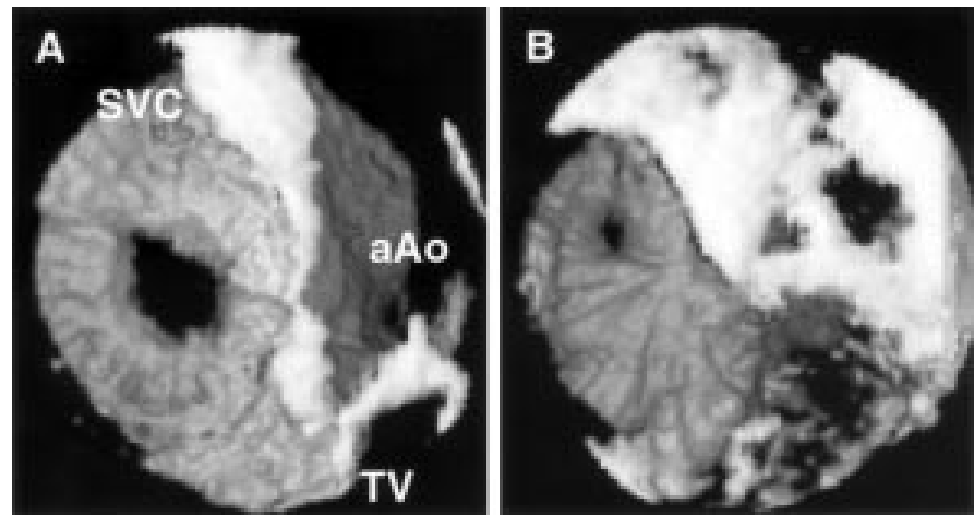

Figure 2 Three dimensional images of an atrial septal defect of largest dimension (panel A) and smallest dimension (panel B). Percentage of change in area in this defect was $86 \%$. aAo, ascending aorta; IVC, inferior vena cava; SVC, superior vena cava. the volume rendered image, the lengths of the major and minor axis were measured on the "anyplane" cross sectional image, reproduced in the respective angles. The area of the defect was calculated from the lengths of the major and the minor axes, assuming an ellipsoidal shape, using the formula: area of the defect $=\pi(R)(r) / 4$, where $R$ is the length of the major axis, and $r$ is the length of the minor axis (fig 1A). On volume rendered images from the largest and smallest dimensions, lengths of the defect parallel and perpendicular to the ascending aorta and to the annulus of the tricuspid valve (fig 1B) were also measured for later calculation of the shortening of the defect from different angles.

When part of the anterior rim of the defect was attenuated (no fold beneath the ascending aorta), the difference in length of the attenuated portion in the frames with the largest defect dimension and the frames with the smallest defect dimension was also measured, using the "anyplane" function. Serial parallel images perpendicular to the aorta were obtained every $0.2-0.3 \mathrm{~mm}$ and the distance between the two outermost planes with no fold beneath the ascending aorta was then measured.

Cross sectional images of both transthoracic and transoesophageal echocardiographic examinations recorded on the same day as the three dimensional echocardiographic study were reviewed, and areas of the defects were measured using off line analysis. The cardiac phases in the largest and smallest dimensions were arbitrarily defined immediately before the opening and closing of the atrioventricular valve, respectively. ${ }^{16}$ For transthoracic images, the sharpest images delineating clear borders of the defect throughout the cardiac cycle were used, regardless of the transducer's position. For the transoesophageal approach, images in the transverse plane corresponding to $0^{\circ}$ rotation were used. The areas of the defect were calculated using lengths from the cross sectional measurements, assuming a circular shape.

The percentage change in the area and length of each measurement during the cardiac cycle was then calculated using the following formula:

$\%$ change during the cardiac cycle $=$ (largest measurement - smallest measurement)/largest measurement $\times 100(\%)$.

STATISTICAL ANALYSIS

Differences between each measurement were compared by a paired $t$ test. Correlations between two measurements were tested by simple linear correlation. Data are presented as mean (SD).

INTEROBSERVER AND INTRAOBSERVER VARIABILITY

In 10 randomly selected patients, the percentage change in defect area was independently measured by two examiners, and repeated by one observer. Interobserver and intraobserver variability was defined as the difference between two measurements, expressed as the 
Table 1 Percentage change in length of the atrial septal defects

\begin{tabular}{lllll}
\hline \multicolumn{5}{l}{ Length of defect $(\mathrm{mm})$} \\
\hline Direction & Largest dimension & Smallest dimension & \% Change & p Value \\
\hline Major axis & $14.5(3.1)$ & $8.9(2.6)$ & $38.3(12.5)$ & \\
Minor axis & $10.8(3.4)$ & $6.6(2.7)$ & $39.0(16.2)$ & 0.73 \\
Parallel to aAo & $13.1(3.4)$ & $8.0(2.6)$ & $38.3(14.9)$ & \\
Perpendicular to aAo & $11.9(3.5)$ & $7.8(2.9)$ & $35.1(17.7)$ & 0.29 \\
& & & & \\
Parallel to TV & $13.3(3.2)$ & $8.4(2.8)$ & $36.3(15.5)$ & \\
Perpendicular to TV & $11.9(3.2)$ & $7.4(2.3)$ & $37.1(14.4)$ & 0.77
\end{tabular}

Values are mean (SD). The $\mathrm{p}$ value represents the differences in percentage change in length between the two directions.

aAo, ascending aorta; TV, tricuspid valve.

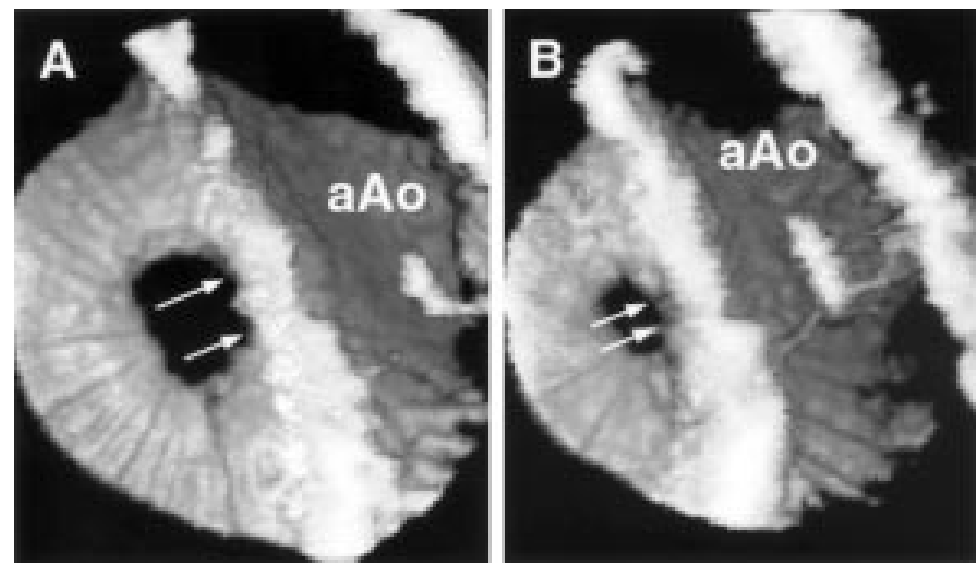

Figure 3 Difference in length of an attenuated anterior rim between largest dimension (panel A) and smallest dimension (panel B). aAo, ascending aorta.

percentage of the average of two values and the standard deviation of the difference of the two values. Interobserver and intraobserver variabilities were $1.2(8.0) \%$ and $1.0(6.6) \%$, respectively.

\section{Results}

DYNAMIC CHANGE IN THE ATRIAL SEPTAL DEFECT The area of the defects changed significantly during the cardiac cycle from $129(61) \mathrm{mm}^{2}$ to 51 (34) $\mathrm{mm}^{2}(\mathrm{p}<0.0001)$ (fig 2). The percentage change ranged from $16.6 \%$ to $86.1 \%$, with a mean of $61.1 \%$. These area changes were found to be unrelated to the direction relative to the axes of the defects or to the ascending aorta or tricuspid annulus. The changes in length between major and minor defect axes $(p=0.73)$, or between the defect dimensions parallel and perpendicular to the ascending aorta or the annulus of the tricuspid valve, were not significantly different $(\mathrm{p}=0.29, \mathrm{p}=0.77$, respectively) (table 1$)$.

In 13 patients with an attenuated anterior rim, the difference in length of the attenuated portion changed significantly during the cardiac cycle, from 54 (36) $\mathrm{mm}$ to 17 (19) $\mathrm{mm}$ $(p=0.001)$ (fig 3). The percentage change in

Table 2 Percentage changes in area of atrial septal defects according to the morphological feature of the defects

\begin{tabular}{llll}
\hline \multicolumn{4}{c}{$\%$ Change in area (mean $(S D))$} \\
\hline Morphological feature & Present & Absent & p Value \\
\hline Aneurysmal septum primum & $63.2(19.3), \mathrm{n}=11$ & $60.4(15.6), \mathrm{n}=30$ & 0.64 \\
Attenuated anterior rim & $67.3(10.9), \mathrm{n}=13$ & $58.2(18.0), \mathrm{n}=28$ & 0.10 \\
\hline
\end{tabular}

this distance ranged from $30.8 \%$ to $100 \%$, mean $70.1 \%$.

\section{POSSIBLE DETERMINANTS OF AMOUNT OF} DYNAMIC CHANGE

The percentage change in the defect area had no correlation with the patients' age $\left(R^{2}=0.00003\right)$, the largest size of the defect $\left(R^{2}=0.006\right)$, heart rate $\left(R^{2}=0.031\right)$, or $\mathrm{Qp} / \mathrm{Qs}$ ratio $\left(R^{2}=0.0002\right)$. In the 11 defects with an associated aneurysmal septum primum, there were no significant differences in the percentages of change in area between those with or without an aneurysmal septum $(\mathrm{p}=0.64)($ table 2$)$. In the 13 defects with an attenuated anterior rim, no difference in the percentage change in the defect area was found between those with and those without an attenuated anterior $\operatorname{rim}(\mathrm{p}=0.10)($ table 2$)$.

TWO DIMENSIONAL ECHOCARDIOGRAPHY

The dimensions of the defects from the frames with the largest and the smallest defect size were measured off line from transthoracic cross sectional images in 29 patients and from transoesophageal cross sectional images in 38 patients. The percentage change in the defect areas obtained from three dimensional echocardiography did not correlate with those obtained from cross sectional images, either by transthoracic echocardiography $\left(R^{2}=0.143\right)$ or by transoesophageal echocardiography $\left(R^{2}=0.148\right)$.

\section{Discussion}

DYNAMIC FEATURES OF ATRIAL SEPTAL DEFECTS Three dimensional echocardiography has recently shown the dynamic nature of the atrial wall with a secundum atrial septal defect, which had not been previously recognised by other imaging techniques. ${ }^{1516}$ We also found that changes in defect areas were quite variable among different patients. This variable change in area may play a role in the natural history of the secundum defect, and may be an important factor when assessing methods of treatment, such as the suitability of transcatheter closure prostheses.

As Franke and colleagues reported, the area of the defect seems to change according to the apical and basal translation of the mitral and tricuspid annuli. ${ }^{16}$ In addition, we found that the changes in defect area were not only parallel to the heart axis from base to apex, but also perpendicular to the axis. Most of the defect areas were reduced concentrically, keeping a similar shape throughout the cardiac cycle. Furthermore, the dynamic, volume rendered three dimensional images showed that there was qualitatively better contractility in the septum secundum region (the rim of the atrial septum) than in the septum primum region (the flap valve).

DETERMINANTS OF VARIABILITY IN CONTRACTION PATTERN

We noted that neither the age of the patient, the size of the defect, the heart rate, nor the amount of volume overload ( $\mathrm{Qp} / \mathrm{Qs}$ ratio) affected the extent of contraction. Although 
Lange and colleagues reported reduced contraction in older patients, ${ }^{15}$ we think the limitations of their transthoracic approach may have been responsible for this discrepancy, as another study using a transoesophageal approach found good contraction, even in an adult population. ${ }^{16}$

ASSESSMENT OF DYNAMIC CHANGES USING TWO DIMENSIONAL IMAGING

Although contraction of the atrial septal defect can be appreciated by cross sectional echocardiography, we showed that the degree of contraction cannot be measured accurately in this way. The change in size of the defect during the cardiac cycle is caused by both defect contraction and heart movement, and these cannot be distinguished by cross sectional echocardiography.

For measuring dynamic changes in defect area, three dimensional echocardiography has two important functions that are lacking with conventional cross sectional echocardiography. First, three dimensional volume rendered images provide the angle of the defect axis. Second, using the "anyplane" function, one can change the plane of the cross sectional image after defining particular segments of the cardiac cycle, such as the phases of the largest or smallest defect size.

\section{CLINICAL IMPLICATIONS}

In previous clinical studies it has been reported that the size of the defect does not correlate with the degree of shunting or with clinical symptoms. ${ }^{4}$ However, our results showed that measurements using cross sectional echocardiography cannot represent the true dynamic area of the defect.

Incremental atrial shunting related to age may be explained not only by the difference in compliance between the left and the right ventricle, ${ }^{20}$ but also by the dynamic features of the defect. Owing to the more rapid heart rate in small children, the period during which the defects are larger-as observed on dynamic three dimensional images - is relatively shorter than in older patients with slower heart rates. In adult patients, left ventricular compliance diminishes with age, ${ }^{21}$ whereas paediatric patients have normal compliance throughout the age range beyond infancy. ${ }^{22}$ Thus heart rate may account for the incremental atrial shunting with age in paediatric patients. In the future, assessment of the dynamic size of atrial defects and the associated flow, using advanced techniques such as semiautomated colour Doppler flow measurement, may further define the clinical status of these patients.

Recently, several devices for transcatheter closure of atrial septal defects have been developed, ${ }^{7-11}$ though all have problems such as complicated deployment or residual leaks. ${ }^{23}{ }^{24}$ A clearer understanding of the dynamic nature of the defects using three dimensional echocardiography may improve the closure rates and the long term cure rates. ${ }^{25} 26$
LIMITATIONS

The three dimensional datasets we used always contain a certain amount of motion artefact, as the serial cross sectional echocardiographic data are acquired during different times in the cardiac cycle. In addition, we calculated the theoretical defect area assuming an ellipsoidal shape, because defect area cannot be measured directly from the three dimensional, volume rendered images. In a previous study, Franke and colleagues used a summation of slices method, ${ }^{16}$ which was modified from measurements of ventricular mass and wall thickness. While this is theoretically reasonable, we found it too difficult to achieve an acceptable degree of agreement between observers owing to the obscured borders of the rim in slices taken at the edge of the defect. Therefore, in agreement with Lange and colleagues, ${ }^{15}$ we used only directly measured major and minor axes, which were more clearly defined by the "anyplane" cross sectional images. Using this calculation, we found relatively high but acceptable ranges for the interobserver and intraobserver variability. In the future, better detection of defect borders using real time three dimensional echocardiography may overcome this limitation. $^{27}$

Inferoposterior atrial septal defects were not clearly defined by three dimensional echocardiography and had to be excluded from this study. These excluded defects may have their own particular pattern of contraction; hence our results may not be applicable to such defects.

\section{CONCLUSIONS}

Three dimensional echocardiography provides new information on the dynamic nature of the secundum atrial septal defect. Most of the defects contracted symmetrically. The amount of contraction varied in each defect, and was not related to the patient's age, defect size, heart rate, Qp/Qs ratio, the presence of an aneurysmal atrial septum, or an attenuated anterior rim. This information could not be obtained by conventional cross sectional echocardiography. Further clinical studies using dynamic three dimensional information may improve the management of patients with secundum atrial septal defects.

This study was supported in part by a research grant from Research Training Committee, the Hospital for Sick Children, Toronto, Canada.

1 Benson LN, Freedom RM. Atrial septal defect. In: Freedom RM, Benson LN, Smallhorn JF, eds. Neonatal heart disease. RM, Benson LN, Smallhorn JF, eds. Neond

2 Ferreira SMAG, Ho SY, Anderson RH. Morphological study of defects of the atrial septum within the oval fossa: implications for transcatheter closure of left-to-right shunt. Br Heart f 1992;67:316-20.

3 Chan KC, Godman MJ. Morphological variations of fossa ovalis atrial septal defects (secundum): feasibility for transcutaneous closure with the clam-shell device. Br Heart $\mathcal{F}$ 1993;69:52-5

4 Tabery S, Daniëls O. How classical are the clinical features of the "ostium secundum" atrial septal defect? Cardiol Young 1997; 7:294-301.

5 Burke RP, Michielon G, Wernovsky G. Video-assisted cardioscopy in congenital heart operations. Ann Thorac Surg 1994;58:864-8.

6 Schwartz DS, Ribakove GH, Grossi EA, et al. Minimally invasive cardiopulmonary bypass with cardioplegic arrest: a invasive cardiopulmonary bypass with cardioplegic arrest: a
closed chest technique with equivalent myocardial protection. $\mathcal{F}$ Thorac Cardiovasc Surg 1996;111:556-66. 
7 Rome JJ, Keane JF, Perry SB, et al. Double-umbrella closure of atrial defects: initial clinical applications. Circulation of atrial defects:

8 Sideris EB, Sideris SE, Thanopoulos BD, et al. Transvenous atrial septal defect occlusion by the buttoned device. Am $\mathcal{F}$ Cardiol 1990;66:1524-6.

9 Das GS, Voss G, Jarvis G, et al. Experimental atrial septal defect closure with a new, transcatheter, self-centering device. Circulation 1993;88:1754-64.

10 Hausdorf G, Schneider M, Franzbach B, et al. Transcatheter closure of secundum atrial septal defects with the atrial septal defect occlusion system (ASDOS): initial experience in children. Heart 1996;75:83-8.

11 Sharafuddin MJA, Gu X, Titus JL, et al. Tranvenous closure of secundum atrial septal defects. Preliminary results with a new self-expanding nitinol prosthesis in a swine model. Circulation 1997;95:2162-8.

12 Shah D, Azhar M, Oakley CM, et al. Natural history of secundum atrial septal defect in adults after medical or surgical treatment: a historical prospective study. Br Heart $f$ 1994;71:224-8

13 Konstantinides S, Geibei A, Olschewski M, et al. A comparison of surgical and medical therapy for atrial septal defect in adults. N Engl F Med 1995;333:469-73.

14 Mügge A, Daniel WG, Angermann C, et al. Atrial septal aneurysm in adult patients. A multicenter study using transthoracic and transesophageal echocardiography. Circulation 1995;91:2785-92.

15 Lange A, Walayat M, Turnbull CM, et al. Assessment of atrial septal defect morphology by transthoracic three dimensional echocardiography using standard gray scale and Doppler myocardial imaging techniques: comparison with magnetic resonance imaging and intraoperative findings. Heart 1997;78:382-9.

16 Franke A, Kühl HP, Rulands D, et al. Quantitative analysis of the morphology of secundum-type atrial septal defect of the dimensional echocardiography. Circulation 1997;96:II323-7.

17 Magni G, Cao Q-L, Sugeng L, et al. Volume-rendered, three-dimensional echocardiographic determination of the size, shape, and position of atrial septal defects: validation in an in vitro model. Am Heart $\mathcal{f}$ 1996;132:376-81.
18 Marx GR, Fulton DR, Pandian NG, et al. Delineation of site, relative size and dynamic geometry of atrial septal defects by real-time three-dimensional echocardiography. $\mathcal{F}$ Am Coll Cardiol 1995;25:482-90.

19 Vogel M, Lösch S, Bühlmeyer K. The application of transthoracic dynamic three-dimensional echocardiography by computer-controlled parallel slicing in patients with fixed subaortic obstruction. Cardiol Young 1994;4:714.

20 Perloff JK. Congenital heart disease in adults. In: Braunwald E, ed. Heart disease. Philadelphia: WB Saunders, 1992:96691.

21 Iwase $M$, Nagata $\mathrm{K}$, Izawa $\mathrm{H}$, et al. Age-related changes in left and right ventricular filling velocity profiles and their relationship in normal subjects. Am Heart f 1993;126:41926

22 Harada K, Suzuki T, Tamura M, et al. Role of age on transmitral flow velocity patterns in assessing left ventricular diastolic function in normal infants and children. $A m \mathcal{F}$ Cardiol 1995;76:530-2.

23 Boutin C, Musewe NN, Smallhorn JF, et al. Echocardiographic follow-up of atrial septal defect after catheter closure by double-umbrella device. Circulation 1993;88: $621-7$.

24 Agarwal SK, Ghosh PK, Mittal PK. Failure of devices used for closure of atrial septal defects: mechanisms and management. F Thorac Cardiovasc Surg 1996;112:21-6.

25 Magni G, Hijazi ZM, Pandian MG, et al. Two- and three-dimensional transesophageal echocardiography in patient selection and assessment of atrial septal defect closure by the new DAS-angel wings device. Circulation 1997; 96:1722-8.

26 Maeno YV, Benson LN, Boutin C. The impact of dynamic three-dimensional transesophageal echocardiography in the assessment of atrial septal defects and occlusion by double-umbrella device (CardioSEAL). Cardiol Young 1998;8:368-78.

27 Shiota $\mathrm{T}$, Jones $\mathrm{M}$, Ota $\mathrm{T}$, et al. Real time 3D echocardiography for assessing the atrial septal defect size and shunting: quantitative studies in a chronic animal and shunting: quantitative studies in a chronic animal
model [abstract]. I Am Coll Cardiol 1998;31(suppl A):259A. 